\title{
Determination of interaction forces between higher plant thylakoids and electron-density-profile evaluation using small-angle $\mathbf{X}$-ray scattering
}

\author{
Kay Diederichs, Wolfram Welte and Werner Kreutz \\ Institut für Biophysik und Strahlenbiologie der Universität Freiburg im Breisgau, Albertstrasse 23, D-7800 Freiburg i.Br. \\ (F.R.G.)
}

Key words: Thylakoid structure; Thylakoid stacking: Small-angle X-ray scattering; Electron density profile; Surface charge (Spinach chloroplast)

Osmotic dehydration of spinach thylakoids has enabled us to calculate the low-resolution (1.0-1.5 $\mathrm{nm})$ electron density profiles of the thylakoids at Bragg periods between 16 and $23 \mathrm{~nm}$. Electron density profile calculation was performed by a least-squares procedure and controlled by mapping the experimental scattering intensities on the continuous Fourier transform of the electron density profiles. The results show that the outsides of opposing thylakoids are appressed closely together, whereas the luminal extent of the thylakoid is a function of the applied osmotic pressure, thus determining the Bragg period. It could be shown that the distance of the inner surfaces of the thylakoid is established by the equilibrium between externally applied osmotic pressure and the electrostatic repulsion at the inner (luminal) surface. The interaction can be modified by the luminal electrolyte concentration. The area per electric charge on the thylakoid inner surface was estimated to be $1.4 \pm 0.2 \mathrm{~nm}^{2}$.

\section{Introduction}

In recent years, much experimental and theoretical work has been devoted to the attractive (Van der Waals) and repulsive (electrostatic, hydration) forces between bilayers. Investigations on neutral egg lecithin (phosphatidylcholine) [1] and charged (various amounts of phosphatidylcholine, phosphatidylglycerol and phosphatidylinositol) bilayers [2] have been described. Many research groups have used small-angle $X$-ray scattering to determine the alteration in the repeat distance of the lamellar phase, composed of alternating layers of water and membranes. Neutron and small-angle $\mathrm{X}$-ray scattering as well as other methods have been used to investigate properties of membranes of photosynthetic plants and algae [3-6]. A major field of study has been the stacking mechanisms of thylakoids in higher plant chloroplasts [7-12]. The electron density profiles of these thylakoids have been found to be asymmetric, which makes it necessary to distinguish between luminal and cytoplasmic interactions.

In this study, we used the theory on the repulsion of charged double layers developed by Ninham and Parsegian [13] to determine the surface charge of the thylakoid inner surface. We investigated the influence of osmotic dehydration on the repeat period of stacked thylakoids using a newly developed osmotic dehydration chamber. Our X-ray data were submitted to detailed analysis using the theory of Welte and Kreutz [14]. This theory takes into account the finite extent and the statistical distortions of the paracrystalline lattice. The results of the electron density profile evaluation indicated that it is relatively easy to distinguish between the luminal and cytoplasmic interactions in spinach chloroplasts because the cy- 
toplasmic interaction is not influenced by external osmotic pressure.

\section{Materials and Methods}

\section{Sample and sample-holder}

Fresh spinach (Spinacia oleracea) chloroplasts were prepared according essentially to the method of Siggel et al. [15]. Spinach leaves were homogenized in a buffer comprising $0.4 \mathrm{M}$ sucrose $/ 2 \mathrm{mM}$ $\mathrm{MgCl}_{2} / 10 \mathrm{mM} \mathrm{NaCl} / 20 \mathrm{mM}$ Tricene/1 mM EDTA/10 $\mathrm{mM}$ sodium ascorbate. They were filtered through a column of nylon sieves, allowing only chloroplasts to pass through. After centrifugation ( $1000 \times \mathrm{g}, 3 \mathrm{~min}$ ), chloroplasts were washed once in $0.4 \mathrm{M}$ sucrose $/ 10 \mathrm{mM} \mathrm{NaCl} / 20 \mathrm{mM}$ Tricene $/ 1 \mathrm{mM}$ EDTA. Subsequently two centrifugations were used to change the buffer to $20 \mathrm{mM}$ Tricene $/ 0.1 \%$ bovine serum albumin $/ 200 \mathrm{mM}$ $\mathrm{NaCl}$. The amounts of salts used ensured that the thylakoids remained in a stacked state [16]. The above preparation yielded a strong, homogeneous band in a sucrose density gradient (35-45 wt.\%). After preparation, the sample (about $100 \mathrm{mg}$ wet pellet) was dialyzed against a sucrose solution of known density containing $20 \mathrm{mM}$ Tricene ( $\mathrm{pH} 7.4$ ) and variable amounts of sodium chloride using a semipermeable membrane (BM1 or BM5, exclusion limit 100 or $500 \mathrm{Da}$, from Berghof, Tübingen, F.R.G.). This dehydration took place in a newly developed, cooled sample-holder (Fig. 1). During $\mathrm{X}$-ray exposure, the sample was contained in a rectangular cavity formed by two mylar X-ray windows on the front and rear face, $1 \mathrm{~mm}$ apart, the dialysis membranes on the top and the bottom face, and the chamber walls on the sides. The lower dialysis membrane was fixed, whereas the upper one could move to allow for the loss of water of the sample. During the dehydration process, water and small ions such as $\mathrm{Na}^{+}$and $\mathrm{Cl}^{-}$ could freely pass the semipermeable membranes, whereas sucrose could not. This applied to the BM5 membrane, with an exclusion limit of 500 $\mathrm{Da}$, as well. The dehydration led to a net orientation of the stacks, because the thickness of the sample was reduced in the vertical direction by a factor of about 5 . The sucrose solution was pumped to and from a reservoir of about $100 \mathrm{ml}$. Its concentration was monitored by using an Abbe

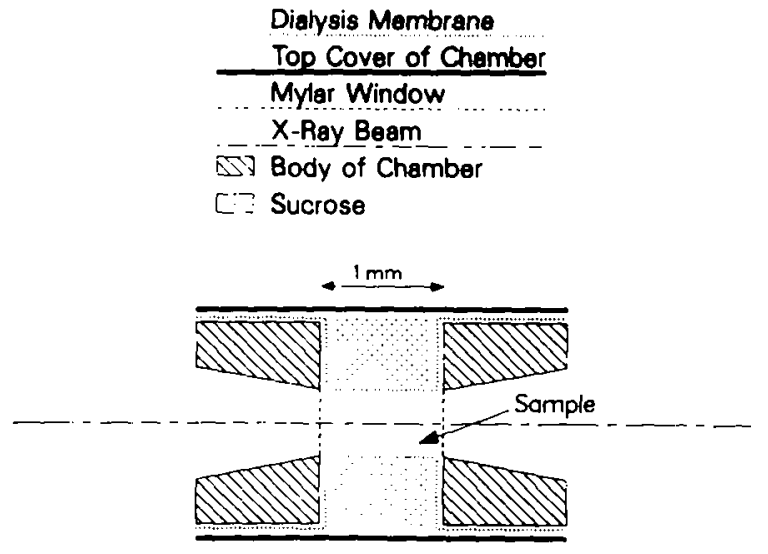

Fig. 1. Schematic drawing of osmotic dehydration chamber.

refractometer (Zeiss). Sucrose was used because: high osmotic pressures (up to $25 \cdot 10^{6} \mathrm{~Pa}$ ) can be obtained with concentrated solutions;

the dehydration is fast compared to the attainment of equilibrium through the vapour phase by means of salt solutions.

All measurements were performed at $8^{\circ} \mathrm{C}$ and the buffers used had a $\mathrm{pH}$ of 7.4.

\section{Small-angle $X$-ray scattering}

Small angle $X$-ray scattering diagrams were recorded using a rotating anode generator $(45 \mathrm{kV}$, $250 \mathrm{~mA}$ ), a Kratky camera and a position-sensitive proportional counter [17]. The cross-section of the primary beam was limited to $100 \times 400 \mu \mathrm{m}$ by additional diaphragms. The specimen to detector distance was $220-240 \mathrm{~mm}$. The diffraction intensity was measured in the direction of the preferred orientation of the membrane stack normals. For the recording of the isotropic background scattering the detector was rotated by $30^{\circ}$ with respect to the direction of the oriented stacks. Recording times ranged from 20000 to $50000 \mathrm{~s}$. The control of the orientation of the stacks was carried out by recording the diffraction pattern on Kodak X-ray films.

\section{Electron-density evaluation method}

After background subtraction, the point focus scattering intensity of the sample was obtained. The electron density profile is the thylakoid electron density projected on an axis perpendicular to the surface of the thylakoid. The electron density profile corresponding to the measured scattering 
was evaluated according to the theory of Welte and Kreutz [14].

The basic features of this theory are outlined briefly in the following. The electron density $\rho(x)$ is expanded into a cosine series:

$\rho(x)=\sum_{k=0}^{N C} c_{k} \cdot \cos (2 \pi k \cdot x / L)$

where $L$ denotes the Bragg period and $N C$ the number of cosine coefficients, $c_{k}$, used to describe the electron density profile.

The procedure predicts the scattering intensity arising from an ensemble of scattering membrane stacks. The predicted scattering intensity is fitted to the experimental intensity by a least-squares fitting program [18]. The measured scattering intensity is fitted with all possible phase combinations of the $c_{k}$. If there are no errors in the measurement of the intensity, the correct phase combination will have a perfect fit.

The following assumptions are made about the sample:

(1) The specimen consists of stacks built up by single membranes with an image-mirror-image sequence. One thylakoid can be considered as an image-mirror pair. The lateral extension of a membrane is large compared with its extension in the direction of the stack axis.

(2) The axes of the membrane stacks possess a distribution of their orientation around a preferential axis ('mosaic spread'). This implies the applicability of the Lorentz correction.

(3) The distance between neighbouring thylakoids fluctuates according to a Gaussian distribution function, $H_{1}$. The distance between image and mirror membrane in a thylakoid (the luminal extent) fluctuates according to a Gaussian distribution function, $H_{2}$, that can be different from $H_{1}$. These distortions of the paracrystalline lattice should be of the second kind, which means that the distance fluctuations do not refer to fixed lattice points but are independent of each other. Overlappings and holes between statistically distributed membranes are corrected by the addition or subtraction of appropriate box functions.

(4) The number, $n$, of thylakoids in a stack fluctuates. We assumed the distribution, $p(n)$, of stack lengths to be Poissonian with mean $a$ :

$p(n)=\left(\mathrm{e}^{-a} \cdot a^{n}\right) / n !$
This assumption is reasonably justified because the sample consists of many stacks, the mean number of thylakoids in a stack is small and the probability that a stack of length $n+1$ is formed from a stack of length $n$ is independent of $n$.

(5) The scattering intensity of the specimen is caused by the incoherent superposition of the individual stack intensities. For details of the theory and the resulting formulae for the scattering intensity, see the original paper.

\section{Model calculations}

The least-squares fitting procedure was submitted to extensive numerical tests. The data for these model calculations were computed by a stand-alone program using a Monte-Carlo method. It turned out that the Fourier coefficients, $c_{k}$, in the cosine expansion of the electron density profile yielded by the least-squares program (assuming a Poisson distribution of stack lengths) are not sensitive against different distributions of stack lengths, as, for instance, box-shaped, triangle-shaped or exponential-shaped distributions. Relative errors in the Fourier coefficients calculated were always less than $10 \%$.

The least-squares procedure always gave the best fit if the $c_{k}$ had the correct phase combination, regardless of the type of length statistics used for calculating the model scattering intensity. The Bragg period is retrieved by the fitting procedure with high accuracy (error less than $0.1 \mathrm{~nm}$ ).

\section{Forces between membranes}

The unspecific forces between bilayers are frequently divided into long-range (Van der Waals attraction, electrostatic repulsion) and short-range (hydration shell repulsion) interactions.

The hydration shells of opposing membranes give rise to a strong, exponentially decreasing repulsion with a decay distance of about $0.2 \mathrm{~nm}$. The nature of this force is not yet fully understood [2]. Cowley et al. [2] could fit their measurements with the following expression for the hydration force, $F_{\mathrm{H}}$

$F_{\mathrm{H}}=10^{10} \cdot \mathrm{e}^{-d / 1.93}[\mathrm{~Pa}]$

where $d$ is measured in angstroms.

Van der Waals forces or dispersion forces occur 
between uncharged bodies through mutual polarization in their molecules. In the case of the 'bilayer geometry', it can be shown that these attractive forces decay with the third power of the distance [19]. We used the expression

$F_{\mathrm{vdW}}=-A /\left(6 \cdot \pi \cdot d^{3}\right)$

assuming a Hamaker constant, $A$, of $5 \cdot 10^{-21} \mathrm{~J}$, which seems to be reasonable for the system concerned [20].

Electrostatic repulsion of charged particles - in the range of $1-10 \mathrm{~nm}$ distance of the bilayers - is the most important of the three forces. It is due to the electrostatic double layers created mainly by lipid polar headgroups (and other charged components of the membrane). This repulsive force also follows an exponential law

$F_{\mathrm{ES}}=\frac{2}{\varepsilon_{\mathrm{r}} \varepsilon_{0}} \cdot \sigma^{2} \cdot \mathrm{e}^{-\mathrm{xd} d}$

where $\sigma$ is the surface charge and $\kappa$ a monotonically growing function of the salt concentration. The distance between the interacting surfaces is denoted by $d$. The formula given above is a good approximation provided that $d>\kappa^{-1}$ [13]. The theory predicts a screening effect on the repulsion at high concentrations of monovalent salt. The same effect is obtained with low concentrations of polyvalent cations.

Fig. 2 shows the three forces mentioned above on a log scale. For comparison, the Van der Waals force (attractive) has been plotted with negative sign.

In equilibrium, the distance of opposed membranes is established by the balance of four forces. Forces tending to decrease membrane separation are the externally applied osmotic pressure, $F_{\mathrm{p}}$, and the Van der Waals force, $F_{\mathrm{vdw}}$. This latter is comparatively strong at very short distances, where it tends to fuse the membranes, and at long distances (above $10 \mathrm{~nm}$ ). Forces increasing membrane separation are the hydration force, $F_{H}$, (strong at small distances) and the electrostatic repulsion, $F_{\mathrm{ES}}$, which is strong at medium distances $(1-10 \mathrm{~nm})$. It is clearly seen from Fig. 2 that each of the internal forces dominates in a certain range of distances, except at very small distances, where it might be difficult to separate

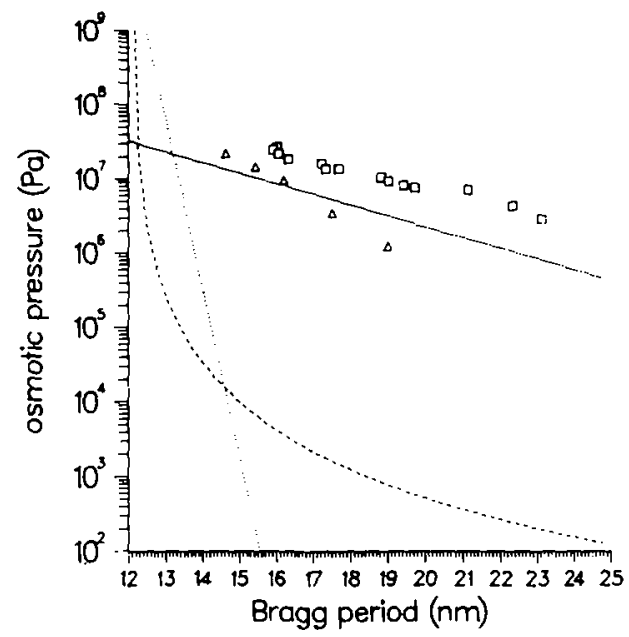

Fig. 2. Comparison of interaction forces and measured data. The zero-point of the interaction forces was assumed to be 12 $\mathrm{nm}$. The $V$ an der Waals force was plotted with negative sign. - electrostatic force, $\sigma=$ one electric charge per $1.5 \mathrm{~nm}^{2}$, $\kappa^{-1}=8 \mathrm{~nm}$. - - - van der Waals force, $A=5 \cdot 10^{-21} \mathrm{~J}$. ..... hydration force, $F_{\mathrm{H}}=10^{10} \cdot \mathrm{e}^{-d / 1.93} \cdot \Delta$, measurements with $100 \mathrm{mM} \mathrm{NaCl}$ in the saccharose solution. $\square$, measurements with $1 \mathrm{mM} \mathrm{NaCl}$ in the saccharose solution.

the influences of the Van der Waals and the hydration force. Data used for the calculation of the curves are given in the legend to Fig. 2.

Although the correct expression for the equilibrium distance, $d$, between bilayers is

$F_{\mathrm{P}}(d)=F_{\mathrm{H}}(d)+F_{\mathrm{FS}}(d)+F_{\mathrm{VdW}}(d)$

we can approximate $F_{\mathrm{P}}$ by $F_{\mathrm{ES}}$ in the range of distances between 3 and $8 \mathrm{~nm}$.

\section{Results}

\section{Scattering diagrams}

Measurements were carried out in a range of sucrose concentrations between 0 and 67 wt. $\%$. Salt concentrations used were $1 \mathrm{mM}$ or $100 \mathrm{mM}$ $\mathrm{NaCl}$. At low sucrose concentrations and under low-salt conditions ( $1 \mathrm{mM} \mathrm{NaCl}$ ), thylakoids tended to swell indefinitely, yielding weak and broad Bragg reflections. Minimum Bragg periods were $15.9 \mathrm{~nm}$ under low-salt conditions and 14.5 $\mathrm{nm}$ under high-salt conditions $(100 \mathrm{mM} \mathrm{NaCl})$. When the salt concentration of the sucrose solution was kept fixed, the Bragg period was a reversible function of the sucrose concentration. 

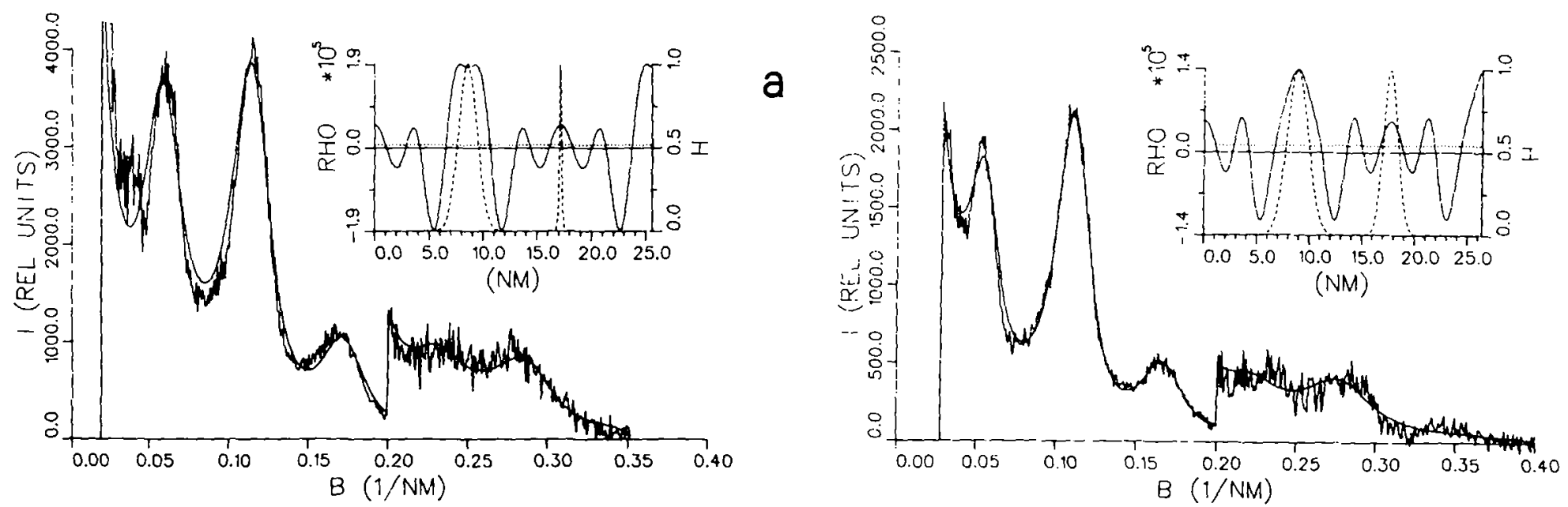

b
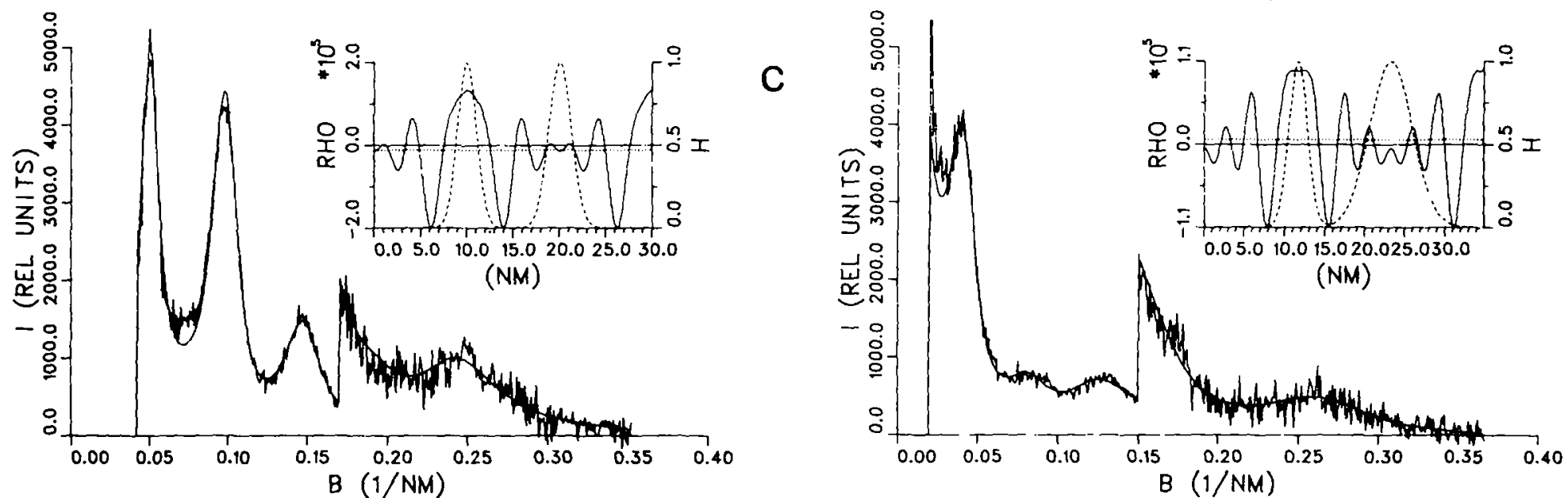

Fig. 3. Comparison of experimental and model intensity for the Bragg periods (a) 17.2 , (b) 17.7 , (c) 20.1 and (d) $23.2 \mathrm{~nm}$. The intensity values for $b>0.2 \mathrm{~nm}$ have been multiplied by 5 . Inset: electron density and statistic functions corresponding to the fit. The horizontal lines indicate solvent level (-_-) and mean electron density $(-\ldots)$. . . 
A plot of our data on a log scale (Fig. 6) shows a linear relationship between Bragg period and sucrose concentration with a slope depending on the $\mathrm{NaCl}$ concentration. As Ninham and Parsegian [13] have shown, this behaviour can be expected if the forces between the thylakoids are interpreted as electrostatic repulsion forces. To determine the surface charge from the measurements, the zerodistance of the force-law has to be known. This is why a detailed analysis of the electron density profile had to be made.

\section{Electron density profile evaluation}

Fits of the electron density were carried out at Bragg periods in the range 16-23 nm. Most of the possible phase combinations could be discarded due to unsatisfactory fits. The few remaining ones had to fulfil the following criteria:

the unit cell should contain the well-known electron density profile of a lipid bilayer

the extension of the unit cell at a large Bragg period compared with the unit cell at a lower Bragg period should be due to an expansion in the inter- or the intrathylakoid space (or both regions).

The only electron density profile that agrees with these criteria is shown in Fig. 3a-d at different Bragg periods, together with the fits and statistic functions $\mathrm{H}_{1}$ and $\mathrm{H}_{2}$. A comparison of cytoplasmic and luminal electron densities suggests that the luminal side of the bilayer is mainly composed of lipids.

The electron densities determined by our

\section{TABLE I}

\section{RELATIVE INTENSITIES}

$n$, order of diffraction; n.d., not determined.

\begin{tabular}{lrrrrrrr}
\hline $\begin{array}{l}\text { Bragg } \\
\text { period } \\
(\mathrm{nm})\end{array}$ & \multicolumn{6}{l}{ Relative intensities } \\
\cline { 2 - 8 } & $n=1$ & $n=2$ & $n=3$ & $n=4$ & $n=5$ & $n=6$ & $n=7$ \\
\hline 15.9 & 45 & 99 & 58 & -51 & -45 & 26 & -19 \\
16.4 & 45 & 97 & 68 & -48 & -50 & 13 & 32 \\
16.8 & 42 & 85 & 71 & -49 & -64 & 17 & 24 \\
17.2 & 48 & 95 & 79 & -32 & -60 & 25 & n.d. \\
17.3 & 42 & 93 & 78 & -33 & -62 & 25 & n.d. \\
17.8 & 36 & 95 & 80 & -18 & -69 & 20 & 20 \\
19.1 & 44 & 113 & 113 & -19 & -79 & 57 & n.d. \\
20.1 & 41 & 93 & 97 & 28 & -74 & -37 & 30 \\
22.7 & 53 & 58 & 98 & 73 & 7 & -82 & -44 \\
\hline
\end{tabular}

method are in good agreement with the measurements of Sadler et al. $[3,4,6]$. See Table I for Fourier coefficients of measurements between 15.9 and $22.7 \mathrm{~nm}$ Bragg period.

The electron density profile shown in Fig. 3 is in good accordance with micrographs showing that the outsides of opposing thylakoids are appressed closely together. A further property of this electron density profile is that the corresponding structure amplitude is zero at $b=0.2 \mathrm{~nm}^{-1}$. This explains a feature common to the measured scattering intensities: they all exhibit an intensity minimum at $b=0.2 \mathrm{~nm}^{-1}$. The structure amplitude corresponding to the cosine coefficients obtained by the fit can be calculated via a Shannon interpolation. Its square agrees well with the visual impression about the course of the structure factor obtained with measurements at different Bragg periods (Fig. 4).

The zero crossing of the structure amplitude at $b=0.2 \mathrm{~nm}^{-1}$ is consistent with a property of the electron density profile: the unit cell obtained by the fits at different Bragg periods is constant. This means that the intercalation of water seems to take place only in that part of the thylakoid which we interpret as the lumen. The opposing outsides of the thylakoid do not seem to alter their distance.

In the following we can therefore assume that a split of the measured Bragg period into two parts is always possible: the lattice unit cell is composed of a constant part of about $12 \mathrm{~nm}$ and a variablewidth part whose extension is a function of the applied osmotic pressure and the concentration of monovalent cations. The constant part represents the so-called partition region (the region where opposing thylakoids touch) composed of the peripheral proteins on the surfaces of the thylakoids and the bilayers forming the lipid matrix of the thylakoid in which intrinsic proteins are immersed. There is an excellent agreement with facts known about the sizes of bilayers with intrinsic proteins: the total thickness of a bilayer is $6 \mathrm{~nm}$, the lengths of the fatty acid chains of lipids amount to $2.8 \mathrm{~nm}$. and the distance between the midpoints of the polar head groups is about $4 \mathrm{~nm}$ [21]. These features of the electron density profile are invariant against osmotic pressure and salt concentration variation, at least at the limited resolution of our experiments (about $1.0-1.5 \mathrm{~nm}$ ). The lumen of the 

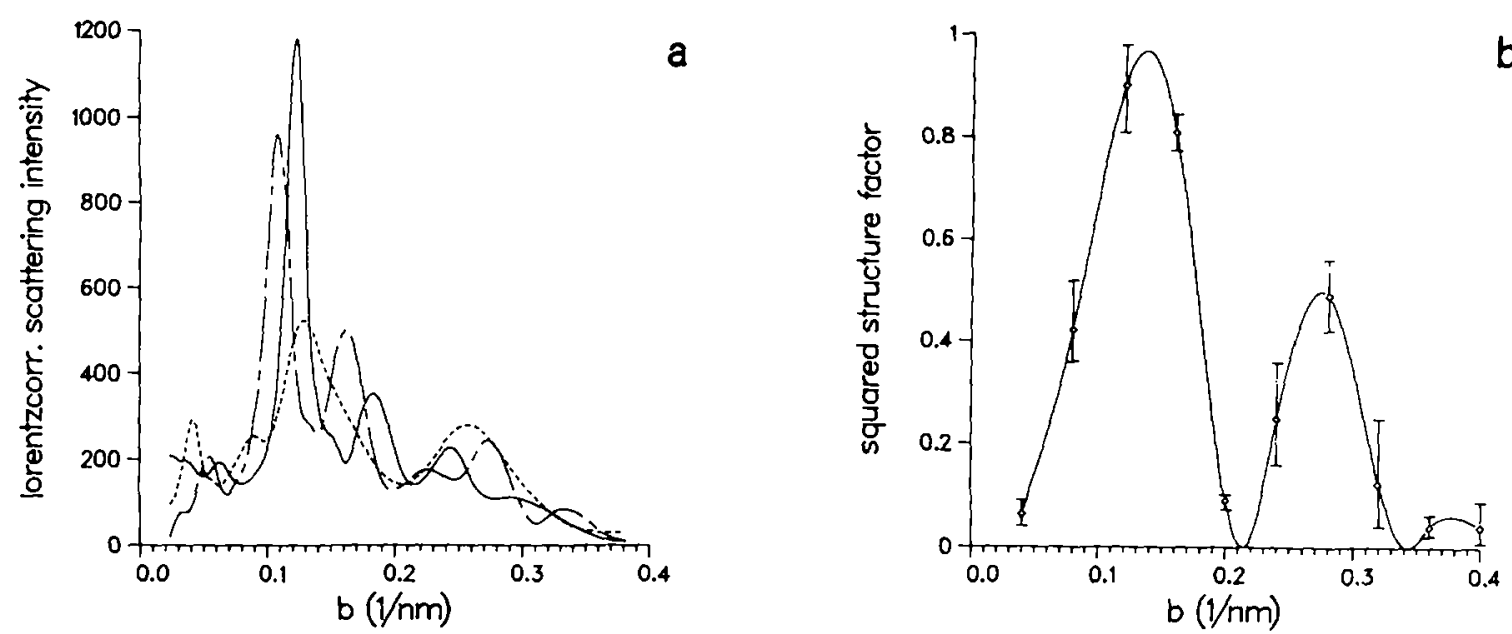

Fig. 4. Comparison between experimental scattering intensities and the squared structure factor corresponding to the calculated electron densities. (a) Scattering intensity of 68 (__ ), 55 ( $\ldots \ldots$ - ) and (35) . . . . -) wt. $\%$ saccharose have been Lorentz-corrected after background subtraction and normalized to the same area under the curve. (b) Mean structure factor from nine fits. Error bars indicate one standard deviation. It is clearly seen that the higher diffraction orders are smeared by disorder. Only the first zero-crossing of the structure factor can be seen in the experimental data.

thylakoid, on the other hand, is variable in its extension and appears to react to the applied osmotic force and salt concentration.

The above-mentioned value of $12 \mathrm{~nm}$ is somewhat arbitrary. Probably, it is an underestimation because of the Helmholtz layer and protruding carbonhydrates that are not seen in the electron density profile (mono- and digalactosyldiacylglycerols form 70\% of the total lipid amount [22]). This value was chosen because we can show, by means of model calculations, that the small variations in the partition region at different Bragg periods as well as the electron density fluctuations in the variable-width part of the thylakoid electron density profile are due to Fourier synthesis artifacts.

Cut-off errors in the Fourier composition of the electron density profile

The error $e(x)$ in the Fourier synthesis $\hat{\rho}(x)$ of the electron density $\rho(x)$ with $N$ coefficients amounts to

$e(x)=\rho(x)-\hat{\rho}(x)=\sum_{k=N+1}^{\infty} c_{k} \cdot \cos (2 \cdot \pi \cdot k \cdot x / L)$

If $\cos (2 \cdot \pi \cdot(N+1) \cdot x / L)$ takes a considerable part in the expansion of $\rho(x)$, we can estimate the magnitude of $e(x)$ by $c_{N+1} \cdot \cos (2 \cdot \pi \cdot(N+1)$. $x / L)$, the first and presumably biggest neglected function in the cosine expansion.

Thus we note the oscillatory character of $e(x)$ and the tendency of large errors at the ends of the expansion interval. Extrema of $e(x)$ are situated at

$x=k \cdot L /(2 \cdot N+2) ; \quad k=0, \ldots, 2 \cdot N+2$

\section{Model calculations}

The fluctuations in the electron density profiles given by the fit were reproduced in a Fourier composition of an artificial step-electron density profile which was synthesized with the same number (6-7) of cosine coefficients as the measured one. The step-model was built up of boxes representing the widths and electron densities known to apply to biological membranes. One half of the model thylakoid is composed of:

a variable-width luminal region accounting for the variation in Bragg period. Its electron density ( 360 $\left.e^{-} / \mathrm{nm}^{3}\right)$ is assumed to be slightly higher than that of pure water $\left(333 e^{-} / \mathrm{nm}^{3}\right)$.

the luminal head group region of the lipids (width $0.8 \mathrm{~nm}$, electron density $\left.450 e^{-} / \mathrm{nm}^{3}\right)$.

a high electron density (450 $\left.e^{-} / \mathrm{nm}^{3}\right)$ partition region made up of lipid headgroups and peripheral 
proteins (wdith $2.1 \mathrm{~nm}$ ).

Fig. 5 demonstrates the excellent agreement between the electron density profiles as yielded by the least-squares procedure and the Fouriercomposed step-profiles. The Fourier-synthesized step profiles show Fourier artifacts that are found in the measured electron density profile in the corresponding Bragg period as well. This pertains to:

fluctuations in the luminal region;

the ripple in the contour of the electron density in the partition region at certain Bragg periods;

the electron density of the polar head-group region on the luminal side of the bilayer which is lower than that of the proteins and the polar headgroups of the lipids on the outer side of the thylakoid. This is, of course, due to the small extension $(0.7-0.8 \mathrm{~nm})$ of the polar headgroups compared with the resolution of $1.0-1.5 \mathrm{~nm}$.

\section{Consequences for the calculation of forces between membranes}

Both the measured and the step-model electron densities suffer from the above mentioned cut-off effects which should be considered in the interpretation of electron density profiles yielded by a Fourier synthesis. We can, therefore, assume a constant part of the thylakoid electron density of a width given by the corresponding step-profile.

Assuming this constant part of about $12 \mathrm{~nm}$, we attribute the luminal variation to the interplay of the electrostatic double layers at the inner surfaces of the bilayers forming a thylakoid. We can thus apply the theory of the diffuse double layer to the lumen of the thylakoid electron density profile. Exponential fits to the measured data show a slope of $\kappa^{-1}=1.5 \mathrm{~nm}$ at $100 \mathrm{mM} \mathrm{NaCl}$ and $\kappa^{-1}=3.66$ $\mathrm{nm}$ at low $\mathrm{NaCl}$ content. Luminal electrolyte concentrations of $40 \mathrm{mM}$ and $7 \mathrm{mM}$ can be calculated from the slopes. The curves cross at a distance of $1.9 \mathrm{~nm}$ from the surface, corresponding to a Bragg period of $13.9 \mathrm{~nm}$ (Fig. 6).

This means that the charges producing the repulsion are apparently located about $1 \mathrm{~nm}$ in front of the bilayer, which can be well explained by the hypothesis of a Helmholtz layer.

If we assume the crossing of the curves: Bragg period vs. osmotic pressure at $100 \mathrm{mM} \mathrm{NaCl}$ and

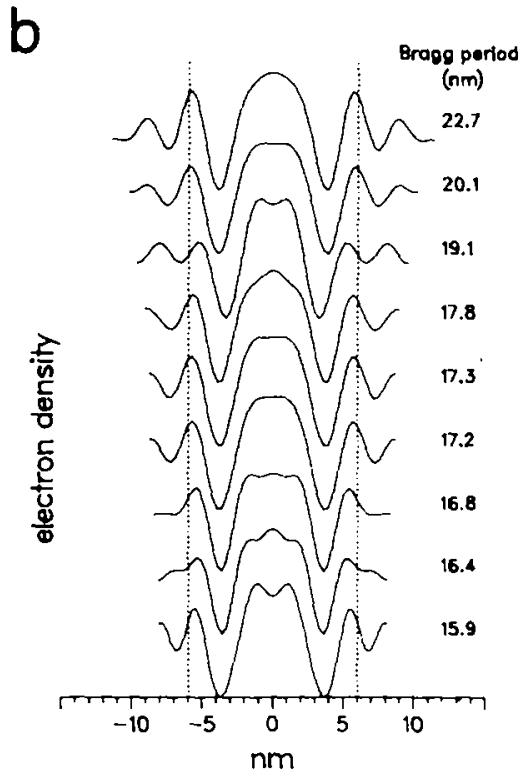

Fig. 5. Comparison of electron density profiles obtained by (a) Fourier-analysis of step-model (see text) and (b) electron density profiles corresponding to fits of experimental scattering intensities at nine different Bragg periods. The vertical dots limit that part of the electron density profile that seems to be constant. This part includes the partition region and the bilayers of opposing thylakoids. It is clearly seen that the extension of the luminal region (outside the dotted lines) determines the Bragg period. Note that the luminal undulations - indicated by arrows in (a) - are similar in both artificial and fitted electron density profiles. The positions of the arrows were calculated according to Eqn. 8. 


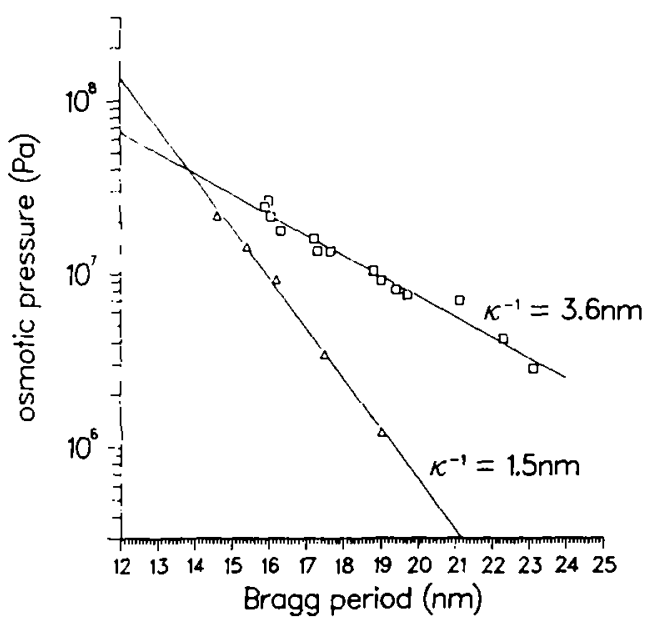

Fig. 6. Exponential fits of the measured data. $\Delta$, measurements with $100 \mathrm{mM} \mathrm{NaCl}$ in the saccharose solution. The slope of the curve corresponds to $40 \mathrm{mM}$ concentration of monovalent cations. $\square$, measurements with $1 \mathrm{mM} \mathrm{NaCl}$ in the saccharose solution. The slope of the curve corresponds to $7 \mathrm{mM}$ concentration of monovalent cations.

Bragg period vs. osmotic pressure at low $\mathrm{NaCl}$ as representing a zero distance between the luminal sides of the bilayers, we can calculate the corresponding surface charge from Eqn. 5 to be 1 electric charge per $1.4 \pm 0.2 \mathrm{~nm}^{2}$. This value has to be compared with the theoretical value calculated from the known lipid composition of the spinach thylakoid bilayer: $30-33 \%$ of the total lipid amount is formed by phospho- and sulfolipids [22,23]. Assuming a surface of about $0.4-0.6 \mathrm{~nm}^{2}$ per lipid, the theoretical values of the surface charge range from 1.2 to $2.0 \mathrm{~nm}^{2}$ per electric charge. Charges from proteins are, however, neglected in this estimation because little is known about the dissociation of protein acidic groups at $\mathrm{pH} 7.4$ and the electron density profiles suggest that the main contribution to the surface charge on the luminal side of the bilayer stems from the lipids.

As we have seen, the electrostatic repulsion between the thylakoid inner surfaces can explain the measured Bragg periods in the range 14.5-23 $\mathrm{nm}$. At larger Bragg periods, the Van der Waals force acquires a magnitude comparable to that of the electrostatic repulsion force. Although our measurements do not cover this range, we can hypothesise that the in vivo equilibrium Bragg period $[3,24]$ of about $25 \mathrm{~nm}$ is due to a balance of the electrostatic repulsion force and the Van der Waals force, influenced perhaps by an osmotic gradient between thylakoid lumen and cytoplasma. Concerning the partition region, our measurements suggest that the surfaces of opposing thylakoids are in contact. We believe that this is due to a complex interaction pattern involving specific protein surface groups and unspecific, attractive forces including the $\mathrm{V}$ an der Waals force.

\section{Acknowledgements}

The authors wish to thank D. Walter and C. Aehnelt for fruitful help and advice. We are grateful to $K$. Bartel and M. Baumstark for many helpful discussions about the investigations, and M. Clarke for correcting the manuscript. All computer calculations were performed at the Rechenzentrum of the University of Freiburg on a Sperry $1100 / 82$.

\section{References}

1 LeNeveu, D.M., Rand, R.P. and Parsegian, V.A. (1976) Nature 259, 601-603

2 Cowley, A.C., Fuller, N.L., Rand, R.P. and Parsegian, V.A (1978) Biochemistry 17, 3163-3168

3 Sadler, D.M. and Worcester, D.L. (1982a) J. Mol. Biol. 159, 467-484

4 Sadler, D.M. and Worcester, D.L. (1982b) J. Mol. Biol. 159. 485-499

5 Hodapp. N. and Kreutz, W. (1980) Biophys. Struct. Mech. 7, 65-95

6 Sadler, D.M., Lefort-tran, M. and Pouphile, M. (1973) Biochim. Biophys. Acta 298, 620-629

7 Barber, J. (1980) Biochim. Biophys. Acta 594, 253-308

8 Rubin, B.T., Chow, W.S. and Barbr, J. (1981) Biochim. Biophys. Acta 634, 174-190

9 Mansfield, R.W., Nakatani, H.Y., Barber, J., Mauro, S. and Lannoye, R. (1982) FEBS Lett. 137 (1), 133-136

10 Albertsson, P. (1982) FEBS Lett. 149 (2), 186-190

11 Duniec, J.T., Israelachvili, J.N., Ninham, B.W., pashley, R.M. and Thorne, S.W. (1981) FEBS Lett. 129 (2), 193-196

12 Duniec, J.T., Sculley, M.J. and Thorne, S.W. (1979) J. Theor. Biol. 79, 473-484

13 Ninham, B. and Parsegian, V.A. (1971) J. Theor. Biol. 31, 405-428

14 Welte, W. and Kreutz, W. (1979) Adv. in Polymer Sci. 30 , 161-225

15 Siggel, U., Renger, G., Stiehl, H.H. and Rumberg, B. (1972) Biochim. Biophys. Acta 256, 328-335

16 Staehelin, L.A. (1976) J. Cell Biol. 71, 136-158

17 Broll, N., Henne, M. and Kreutz, W. (1979) Siemens Analytical Application, Note No. 271 
18 Marquardt, D.W. (1963) J. Soc. Indust. Appl. Math. 2 , 431-441

19 Mahanty, J. and Ninham, B. (1976) Dispersion Forces, pp. 10-13, Academic Press, New York

20 Sculley, M.J., Duniec, J.T., Thorne, S., Chow, W.S. and Boardman, N.K. (1980) Arch. Biochem. Biophys. 201(1), $339-346$
21 Worthington, C.R. and McIntosh, T.J. (1974) Biophys. J. 14, 703-729

22 Allen, C.F., Good, P., Trosper, T. and Park, R.B. (1972) Biochim. Biophys. Res. Commun. 48, 907-913

23 Nishinara, M., Yokota, K. and Kito, M. (1980) Biochim. Biophys. Acta 617, 12-19

24 Kreutz, W. (1964) Z. Naturforsch. 19b, 441-446 